\title{
The Statistical Evaluation Ofcoefficients, Characterizing Unsinusoidality and Unsymmetry of Feed Voltage in Private Housing Projects' Power Supply Systems
}

\author{
Michael A. Averbukha, \\ Eugene V. Zhilin a and Evgenia Yu. Sizganova*b \\ ${ }^{a}$ Belgorod State Technological University named after V.G. Shukhov \\ 46 Kostyukova Str., Belgorod, 308012, Russia \\ ${ }^{b}$ Siberian Federal University \\ 79 Svobodny, Krasnoyarsk, 660041, Russia
}

Received 12.09.2017, received in revised form 09.10.2017, accepted 02.12.2017

The article presents a statistical analysis of the alteration of coefficients, which characterize the unsinusoidality and unsymmetry of the feed voltage and the utilized current in the electrical power supply systems of private housing projects (PHP). It is shown that, depending on the time of day, we can single out three characteristical periods in the daily schedule of the power, consumed by the private housing projects' current-using equipment. The experiments have proved that the coefficients alteration refers to random processes with the pronounced trend and harmonic components. By means of calculation the regular it ies of random processes' numerical characteristic salterations were received in time function for each characteristical period. Based on the seformulas, the most probable values of the coefficients are determined, with account of $5 \%$ annual loads growth. These values allow evaluating the power waste in private housing projects'power supply systems and select the engineering solutions for their reduction.

Keywords: higher harmonic components of currents and voltages, coefficients, characterizing the unsinusoidality and unsymmetry, random process, mathematical expectation and random-process dispersion, correlation coefficient, confidential probability, fitting criteria, confidence intervals.

Citation: Averbukh M.A., Zhilin E.V., Sizganova E.Yu. The statistical evaluation ofcoefficients, characterizing unsinusoidality and unsymmetry of feed voltage in private housing projects' power supply systems, J. Sib. Fed. Univ. Eng. technol., 2017, 10(8), 1079-1087. DOI: 10.17516/1999-494X-2017-10-8-1079-1087.

(C) Siberian Federal University. All rights reserved

* Corresponding author E-mail address: avers45@rambler.ru, seu_eset@mail.ru 


\title{
Статистическая оценка коэффициентов, характеризующих несинусоидальность и несимметрию питающего напряжения в системах электроснабжения ИЖС
}

\author{
М.А. Авербух ${ }^{\mathbf{a}}$, Е.В. Жилин ${ }^{\mathrm{a}}$, Е.Ю. Сизганова ${ }^{\mathbf{\sigma}}$ \\ ${ }^{a}$ Белгородский государственный технологический университет \\ им. В.Г. Шухова \\ Россия, 308012, Белгород, ул. Костюкова, 46 \\ ${ }^{6}$ Сибирский федеральный университет \\ Россия, 660041, Красноярск, пр. Свободный, 79
}

В статье приводится статистический анализ изменения коэффициентов, характеризующих несинусоидальность и несимметрию питающего напряжения и потребляемого тока 6 системах электроснабжения индивидуального жилищного строительства (ИЖС). Показано, что в зависимости от времени суток можно выделить три характерных периода в суточном графике потребляемоймощности электроприемниками ИЖС. Экспериментами установлено, что изменения коэффициентов относятся к случайным проиессам с явно выраженным трендом и гармоническими составляющими. Рассчитаны законы изменения числовых характеристик случайного процесса в функиии времени для каждого характерного периода. На основании этих выражений определяются наиболее вероятные значения коэффициентов с учетом ежегодного роста нагрузок на $5 \%$. Эти значения позволяют оценить потери энергии в системах ИЖС и выбрать технические решения для их снижения.

Ключевые слова: высшие гармонические составляюшие токов и напряжений, коэффициенты, характеризующие несинусоидальность и несимметрию, случайный процесс, математическое ожидание и дисперсия случайного проиесса, коэффиџиент корреляиии, доверительная вероятность, критерии согласия, доверительные интервальл.

В современных системах электроснабжения ИЖС, питающих коммунально-бытовые электроприемники, нелинейную вольтамперную характеристику имеют следующие электроприборы: газоразрядные лампы (энергосберегающие лампы); установки дуговой и контактной сварки; приборы с преобразователями переменного тока в постоянный; системы бесперебойного питания; импульсные источники питания (персональные компьютеры, телевизоры аудиосистемы); преобразователи частоты (СВЧ печи); двигатели с регулируемой скоростью вращения (дрели, стиральные машины, пылесосы), которые являются источниками высших гармоник тока и напряжения, генерируемых в систему электроснабжения. Кроме этого, все перечисленные электроприемники питаются от однофазной сети, что приводит к несимметрии токов и напряжений в четырехпроводной трехфазной системе электроснабжения ИЖС и протеканию тока небаланса в нулевом проводе [1]. При этом спрос на электроэнергию в системах электроснабжения ИЖС носит случайный характер, что связано со временем суток, с рабочими и выходными днями и временами года. В течение дня можно выделить три характерных периода, связанных с началом рабочего дня, интенсивной работой в течение дня и вечерним отдыхом. Изменение спроса электроэнергии в зависимости от времени относится к случайным процессам. Определения числовых характеристик слу- 
чайных процессов базируются на вероятностных методах. Основанием для этого служат экспериментальные данные.

Экспериментальные исследования проводили при помощи сертифицированных приборов «Энергомонитор - 3.3T1» и C.A 8335 (Qualistar +) в течение одной недели, со временем усреднения десять минут [2]. Фрагмент результатов измерения за одни сутки для характерных периодов суток, усредненных для трех фаз, представлен в табл. 1.

Математическая модель процесса со случайным отклонением суммарного коэффициента гармонических составляющих тока может быть представлена в виде соотношения:

$$
K_{I i}=\varphi\left(K_{I i}\right)+\Delta_{i},
$$

где $K_{I i}$ - величина, отражающая ряд наблюдений суммарного коэффициента гармонических составляющих тока $(i=1,2, \ldots, 24) ; \varphi\left(K_{I i}\right)$ - некоторая детерминированная функция, отображающая общую тенденцию изменения $K_{l i}$ (тренд); $\Delta_{i}$ - случайные отклонения, имеющие место при протекании процесса $K_{I i}[3]$.

Для установления соответствия изменения случайной величины, суммарного коэффициента гармонических составляющих тока, в виде случайного процесса определяется корреляционная связь между сечениями для двух значений характерных периодов суток времени $t_{\mathrm{i}}$ и $t_{\mathrm{i}+1}$.

С этой целью устанавливают основные количественные характеристики случайного процесса: математическое ожидание сечения $m_{K i}(t)$, дисперсию $D_{K i}(t)$ сечения, коэффициенты корреляции между сечениями $p_{K i}\left(t_{i}, t_{i+1}\right)$.

Таблица 1

Table 1

\begin{tabular}{|c|c|c|c|c|c|c|c|c|}
\hline \multirow{2}{*}{\multicolumn{2}{|c|}{$\begin{array}{c}\text { Характерные периоды } \\
\text { суток, ч }\end{array}$}} & \multicolumn{7}{|c|}{ Номера суток за неделю } \\
\hline & & \multirow{2}{*}{$\begin{array}{c}1 \\
13,32\end{array}$} & \multirow{2}{*}{$\begin{array}{c}2 \\
13,87\end{array}$} & \multirow{2}{*}{$\begin{array}{c}3 \\
14,71\end{array}$} & \multirow{2}{*}{$\begin{array}{c}4 \\
12,88\end{array}$} & \multirow{2}{*}{$\begin{array}{c}5 \\
13,12\end{array}$} & \multirow{2}{*}{$\begin{array}{c}6 \\
11,99\end{array}$} & \multirow{2}{*}{$\begin{array}{c}7 \\
12,05\end{array}$} \\
\hline & $K_{I}, \%$ & & & & & & & \\
\hline \multirow{3}{*}{$6: 00-9: 00$} & $K_{U}, \%$ & 2,86 & 3,11 & 3,23 & 3,12 & 3,43 & 2,76 & 2,68 \\
\hline & $K_{2 U}, \%$ & 0,45 & 0,52 & 0,48 & 0,46 & 0,51 & 0,47 & 0,42 \\
\hline & $K_{0 U}, \%$ & 1,86 & 2,14 & 2,13 & 1,89 & 1,92 & 1,87 & 1,85 \\
\hline \multirow{4}{*}{$18: 00-21: 00$} & $K_{I}, \%$ & 18,71 & 17,45 & 18,66 & 17,61 & 17,86 & 16,23 & 15,82 \\
\hline & $K_{U}, \%$ & 3,96 & 4,13 & 4,23 & 4,21 & 4,16 & 3,86 & 3,46 \\
\hline & $K_{2 U}, \%$ & 0,76 & 0,67 & 0,82 & 0,73 & 0,65 & 0,66 & 0,52 \\
\hline & $K_{o U}, \%$ & 4,23 & 4,32 & 4,21 & 3,86 & 5,1 & 4,11 & 4,32 \\
\hline \multirow{4}{*}{$23: 00-2: 00$} & $K_{I}, \%$ & 9,44 & 9,77 & 10,43 & 10,67 & 10,31 & 9,06 & 9,02 \\
\hline & $K_{U}, \%$ & 2,32 & 2,45 & 2,44 & 2,67 & 2,43 & 2,12 & 2,31 \\
\hline & $K_{2 U}, \%$ & 0,43 & 0,38 & 0,42 & 0,46 & 0,45 & 0,41 & 0,42 \\
\hline & $K_{0 U}, \%$ & 0,9 & 1,23 & 1,13 & 0,97 & 1,17 & 0,92 & 0,86 \\
\hline
\end{tabular}

Примечание: $K_{I}$ и $K_{U}$ - суммарные коэффициенты гармонических составляющих тока и напряжения (\%); $K_{2 U}$ и $K_{0 U}$ - коэффициенты несимметрии напряжений по обратной и нулевой последовательности (\%). 
Математическое ожидание для сечения $t_{k}$ :

$$
m_{K i}\left(t_{i}\right)=\sum_{i=1}^{n} \frac{K_{I i}\left(t_{i}\right)}{n}
$$

где $\mathrm{n}=7$ - количество суток в неделю.

Дисперсия для сечения $t_{i}$ :

$$
D_{\mathrm{Ki}}\left(t_{k}\right)=\sum_{i=1}^{n} \frac{\left(K_{i}\left(t_{i}\right)-m_{K i}\left(t_{i}\right)\right)^{2}}{n-1} .
$$

Корреляционный коэффициент для сечения $t_{i}$ и $t_{i+1}$ :

$$
\rho_{K i}\left(t_{k}, t_{l}\right)=\frac{\sum_{i=1}^{n}\left(K_{I}\left(t_{i}\right)-m_{K i}\left(t_{i}\right)\right) \cdot\left(K_{I}\left(t_{i+1}\right)-m_{K i}\left(t_{i+1}\right)\right)}{(n-1) \cdot \delta_{K i}\left(t_{i}\right) \cdot \delta_{K i}\left(t_{i+1}\right)},
$$

где $\delta_{\text {кі }}(\mathrm{t})$ - стандартное отклонение.

Результаты расчетов количественных характеристик случайного процесса, для остальных коэффициентов качества электроэнергии вычисляют аналогично (табл. 2).

Как следует из табл. 2, значения корреляционных коэффициентов указывают на то, что изменения коэффициентов качества электроэнергии в течение суток и недели соответствуют стационарным случайным процессам. При этом отклонения от тренда изменения коэффициентов качества электроэнергии во времени производятся путем интерполяции и экстраполяции в соответствии с выражением [4]

$$
m_{K i}(t)=\alpha_{0}+\sum_{k=1}^{p}\left(\alpha_{k} \cdot \cos \frac{2 \pi k t}{n+1}+\beta_{k} \cdot \sin \frac{2 \pi k t}{n+1}\right)+\mu \cdot \omega_{1}(t),
$$

где $p$ - число характерных периодов за сутки; $\alpha_{0}, \alpha_{k}, \beta_{k}, \mu, \omega_{1}(t)$ - коэффициенты, определяющиеся по формулам:

\begin{tabular}{|c|c|c|c|c|c|c|c|c|c|c|c|c|}
\hline \multirow{2}{*}{ № } & \multicolumn{4}{|c|}{1} & \multicolumn{4}{|c|}{2} & \multicolumn{4}{|c|}{3} \\
\hline & $K_{I}, \%$ & $K_{U}, \%$ & $K_{2 U}, \%$ & $K_{O U}, \%$ & $K_{I}, \%$ & $K_{U}, \%$ & $K_{2 U}, \%$ & $K_{o U}, \%$ & $K_{I}, \%$ & $K_{U}, \%$ & $K_{2 U}, \%$ & $K_{o U}, \%$ \\
\hline $\mathrm{m}_{\mathrm{k}}\left(\mathrm{t}_{\mathrm{i}}\right)$ & 13,14 & 3,03 & 0,47 & 1,95 & 17,48 & 4,01 & 0,69 & 4,31 & 9,81 & 2,39 & 0,42 & 1,03 \\
\hline $\mathrm{D}_{\mathrm{k}}\left(\mathrm{t}_{\mathrm{i}}\right)$ & 0,94 & 0,07 & 0,001 & 0,02 & 1,23 & 0,08 & \begin{tabular}{|l|}
0,009 \\
\end{tabular} & 0,15 & 0,45 & 0,03 & 0,001 & 0,02 \\
\hline $\mathrm{p}_{\mathrm{Ki}}\left(\mathrm{t}_{\mathrm{i}}, \mathrm{t}_{\mathrm{i}+1}\right)$ & \multicolumn{4}{|c|}{0,82} & \multicolumn{4}{|c|}{0,63} & \multicolumn{4}{|c|}{0,61} \\
\hline $\mathrm{p}_{\mathrm{Ku}}\left(\mathrm{t}_{\mathrm{i}}, \mathrm{t}_{\mathrm{i}+1}\right)$ & \multicolumn{4}{|c|}{0,84} & \multicolumn{4}{|c|}{0,61} & \multicolumn{4}{|c|}{0,62} \\
\hline $\mathrm{p}_{\mathrm{K} 2 \mathrm{u}}\left(\mathrm{t}_{\mathrm{i}}, \mathrm{t}_{\mathrm{i}+1}\right)$ & \multicolumn{4}{|c|}{0,28} & \multicolumn{4}{|c|}{0,15} & \multicolumn{4}{|c|}{$-0,27$} \\
\hline $\mathrm{p}_{\mathrm{K} 0 \mathrm{u}}\left(\mathrm{t}_{\mathrm{i}}, \mathrm{t}_{\mathrm{i}+1}\right)$ & \multicolumn{4}{|c|}{0,032} & \multicolumn{4}{|c|}{0,471} & \multicolumn{4}{|c|}{0,823} \\
\hline
\end{tabular}

$$
\alpha_{0}=\frac{1}{n+1} \sum_{i=0}^{n} K_{I i}, \quad \alpha_{k}=\frac{2}{n+1} \sum_{i=0}^{n} K_{I i} \cdot \cos \frac{2 \pi k t}{n+1},
$$

Таблица 2

Table 2 


$$
\begin{aligned}
& \beta_{0}=\frac{2}{n+1} \sum_{i=0}^{n} K_{I i} \cdot \sin \frac{2 \pi k t}{n+1}, \quad \mu=\frac{\sum_{i=1}^{n} K_{I i} \cdot \omega_{1}(t)}{\sum_{i=0}^{n} \omega_{1}^{2}(t)}, \\
& \omega_{1}(t)=t-\frac{n}{2}+\sum_{k=1}^{p} \cos \frac{2 \pi k t}{n+1}-\frac{2}{n-1} \sum_{k=1}^{p}\left(\sum_{t=0}^{n} \sin \frac{2 \pi k t}{n+1}\right) \sin \frac{2 \pi k t}{n+1} .
\end{aligned}
$$

Аналитические выражения, полученные на основании экспериментальных значений (за двое суток), при $p=3$ для коэффициентов качества электроэнергии примут следующий вид: суммарный коэффициент гармонических составляющих тока $\left(K_{I}\right)$

$$
\begin{aligned}
& m_{K i}(t)=13,29+0,029 t+0,301 \cos \frac{2 \pi t}{49}-1,26 \cos \frac{4 \pi t}{49}+0,094 \cos \frac{6 \pi t}{49}+0,74 \sin \frac{2 \pi t}{49}- \\
& -1,08 \sin \frac{4 \pi t}{49}-0,62 \sin \frac{6 \pi t}{49} ;
\end{aligned}
$$

суммарный коэффициент гармонических составляющих напряжения $\left(K_{U}\right)$

$$
\begin{aligned}
& m_{K u}(t)=3,308+0,024 t+0,027 \cos \frac{2 \pi t}{49}+0,318 \cos \frac{4 \pi t}{49}+0,048 \cos \frac{6 \pi t}{49}- \\
& -0,063 \sin \frac{2 \pi t}{49}-0,667 \sin \frac{4 \pi t}{49}+0,084 \sin \frac{6 \pi t}{49}
\end{aligned}
$$

коэффициент несимметрии напряжения по обратной последовательности $\left(K_{2 U}\right)$

$$
\begin{aligned}
& m_{K_{2 U}}(t)=0,587-0,00089 t-0,007 \cos \frac{2 \pi t}{49}+0,015 \cos \frac{4 \pi t}{49}-0,037 \cos \frac{6 \pi t}{49}+ \\
& +0,021 \sin \frac{2 \pi t}{49}+0,066 \sin \frac{4 \pi t}{49}+0,0044 \sin \frac{6 \pi t}{49}
\end{aligned}
$$

коэффициент несимметрии напряжения по нулевой последовательности $\left(K_{0 U}\right)$

$$
\begin{aligned}
& m_{K_{0 U}}(t)=1,363+0,008 t+0,124 \cos \frac{2 \pi t}{49}+0,491 \cos \frac{4 \pi t}{49}-0,071 \cos \frac{6 \pi t}{49}- \\
& -0,108 \sin \frac{2 \pi t}{49}-0,283 \sin \frac{4 \pi t}{49}+0,186 \sin \frac{6 \pi t}{49} .
\end{aligned}
$$

Экспериментальные значения и аналитические приближения трендов коэффициентов качества электроэнергии представлены на рис. 1.

Статистическая значимость в целом оценки регрессий определяется по критерию Фишера:

$$
F=\frac{n-l}{l} \cdot \frac{\sum_{i=1}^{n}\left(\bar{K}_{I i}-\bar{K}_{I}\right)^{2}}{\sum_{i=1}^{n}\left(K_{I i}-\bar{K}_{I i}\right)^{2}},
$$


где $\bar{K}_{I}=\frac{1}{n+1} \cdot \sum_{i=0}^{n} K_{I i}-$ среднее значение; $l$ - число искомых параметров тренда без учета постоянной составляющей.

Уравнение регрессии в целом считается значимым с уровнем значимости $\alpha$ (соответственно, доверительная вероятность $q=1-\alpha)$, если значение статистически удовлетворяет неравенству:

$$
F>F_{l-\alpha}(l, n-l)
$$

В практических расчетах широкое употребление получил 5\%-ный уровень значимости $(\alpha=0,05)$.

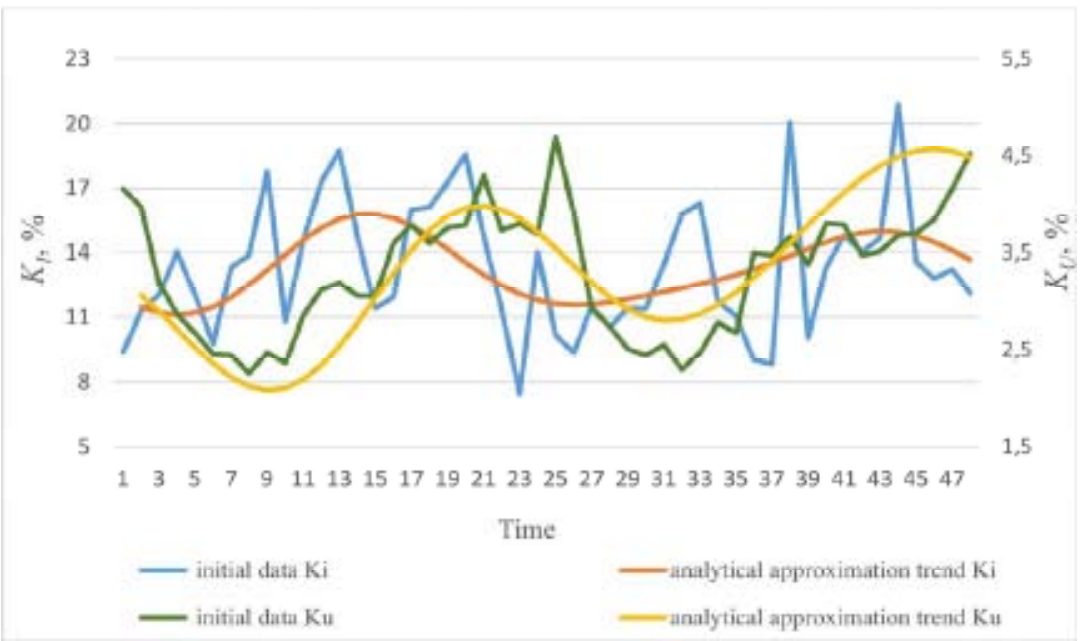

a)

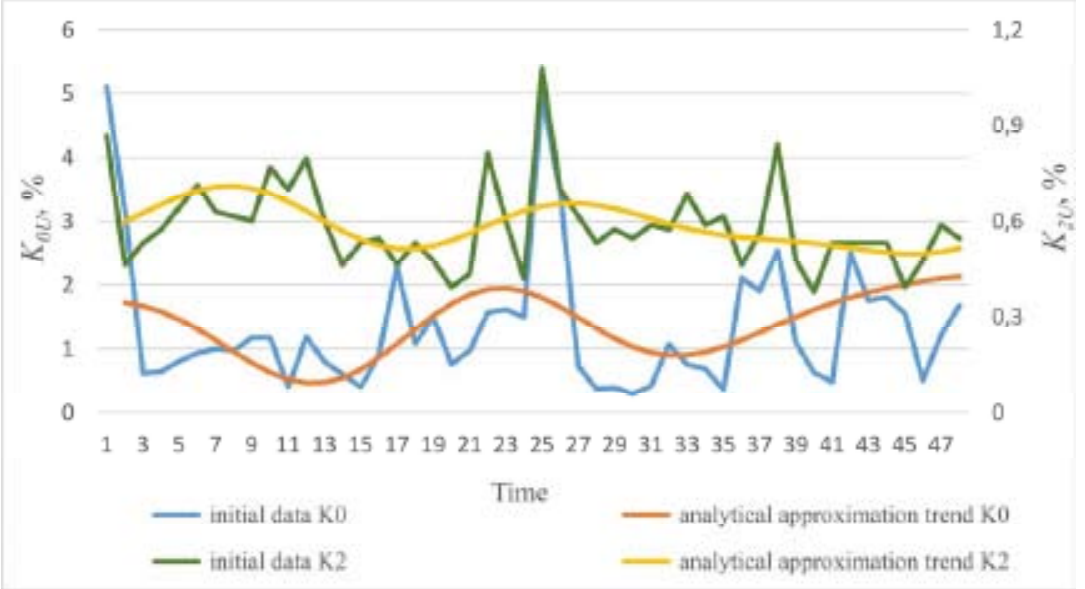

б)

Рис. 1. Тренды коэффициентов качества электроэнергии: a - суммарных коэффициентов гармонических составляющих тока и напряжения; б - несимметрии напряжения по обратной и нулевой последовательности

Fig. 1. Energy quality coefficients trends: a) total harmonic distortion current and voltage components; b) voltage unbalance by reverse and zero sequence 
Проверка на значимость тренда показала выполнение вышеуказанного неравенства при 5\%-ном уровне, т.е. можно утверждать, что с высокой вероятностью полученные оценки трендов не находятся в явном противоречии со статическими данными в период наблюдения.

Полученные аналитические зависимости позволяют прогнозировать показатели качества электроэнергии в системах электроснабжения ИЖС. Задача прогноза заключается в оценке возможной величины значений коэффициентов качества электроэнергии, выходящих за пределы измерений, с учетом ежегодного роста нагрузок на $5 \%$. По данным результатов аналитического приближения тренда определен точечный прогноз значений коэффициентов качества электроэнергии на следующий год. Для повышения достоверности прогноза необходимо по заданной доверительной вероятности построить соответствующий доверительный интервал для аналитического приближения тренда. Границы доверительного интервала для суммарного коэффициента гармонических составляющих тока будут находиться в пределах:

$$
\begin{aligned}
& z_{1}(t) \leq Z_{k i}(t) \leq z_{1}(t), \\
& z_{k}=m_{K i}(t) \pm \sigma\left[m_{K i}(t)\right] \cdot t_{\frac{1+q}{2}}(n-2 p-1),(\mathrm{k}=1,2),
\end{aligned}
$$

где $q$ - доверительная вероятность; $t_{y}(n-2 p-1)$ - квантиль порядка $\gamma$ распределения Стьюдента с $n-2 p-1$ степенями свободы [4].

Определение квантиля производится по таблице 3 [5], входом в которую служат числа $\gamma=0,05$ и $n-2 p-1$. Отсюда следует, что $t_{\frac{1+q}{2}}(n-2 p-1)=2,06$.

В табл. 3 представлены значения для суммарного коэффициента гармонических составляющих тока в условно прогнозируемые сутки на последующий год и границы 95\%-ных симметричных доверительных интервалов.

Аналитическое выражения тренда, включающее в себя показатели всех анализируемых коэффициентов качества электроэнергии за третьи сутки и содержащее 72 значения согласно вышепредставленным формулам, при $p=3$ примет следующий вид:

суммарный коэффициент гармонических составляющих тока $\left(K_{I}\right)$ :

$$
\begin{aligned}
& m_{K i}(t)=13,12+0,000085 t-0,0153 \cos \frac{2 \pi t}{73}-0,665 \cos \frac{4 \pi t}{73}-1,627 \cos \frac{6 \pi t}{73}+ \\
& +0,064 \sin \frac{2 \pi t}{73}+0,528 \sin \frac{4 \pi t}{73}-0,881 \sin \frac{6 \pi t}{73}
\end{aligned}
$$

суммарный коэффициент гармонических составляющихнапряжения $\left(K_{U}\right)$ :

$$
\begin{aligned}
& m_{K u}(t)=3,323+0,0098 t-0,0707 \cos \frac{2 \pi t}{73}-0,1005 \cos \frac{4 \pi t}{73}+0,0899 \cos \frac{6 \pi t}{73}- \\
& -0,1308 \sin \frac{2 \pi t}{73}-0,078 \sin \frac{4 \pi t}{73}-0,667 \sin \frac{6 \pi t}{73}
\end{aligned}
$$

коэффициент несимметрии напряжения по обратной последовательности $\left(K_{2 U}\right)$ :

$$
-1085-
$$


Таблица 3

Table 3

\begin{tabular}{|c|c|c|c|c|c|}
\hline Часы & $\begin{array}{c}\text { Суммарный } \\
\text { коэффициент } \\
\text { гармонических } \\
\text { составляющих тока, } K_{I}\end{array}$ & $\begin{array}{c}\text { Значение } \\
\text { тренда } m_{K i}(t)\end{array}$ & $\begin{array}{c}\text { Средне- } \\
\text { квадратичное } \\
\text { отклонение } \sigma\left[m_{K i}(t)\right]\end{array}$ & $\begin{array}{c}\text { Граница } \\
\text { доверительного } \\
\text { интервала } z_{1}(t)\end{array}$ & $\begin{array}{c}\text { Граница } \\
\text { доверительного } \\
\text { интервала } z_{2}(t)\end{array}$ \\
\hline $0: 00$ & 9,90 & 12,38 & 1,047 & 10,22 & 14,54 \\
\hline $1: 00$ & 11,03 & 12,19 & 1,074 & 9,98 & 14,40 \\
\hline $2: 00$ & 14,48 & 12,08 & 1,129 & 9,76 & 14,41 \\
\hline $3: 00$ & 13,28 & 12,08 & 1,170 & 9,66 & 14,49 \\
\hline $4: 00$ & 13,04 & 12,16 & 1,173 & 9,75 & 14,58 \\
\hline 5:00 & 12,60 & 12,34 & 1,155 & 9,96 & 14,72 \\
\hline $6: 00$ & 12,85 & 12,60 & 1,152 & 10,23 & 14,98 \\
\hline $7: 00$ & 10,38 & 12,92 & 1,172 & 10,50 & 15,33 \\
\hline $8: 00$ & 10,80 & 13,27 & 1,190 & 10,81 & 15,72 \\
\hline 9:00 & 13,46 & 13,62 & 1,189 & 11,17 & 16,07 \\
\hline $10: 00$ & 11,58 & 13,95 & 1,176 & 11,53 & 16,38 \\
\hline $11: 00$ & 15,76 & 14,24 & 1,161 & 11,85 & 16,63 \\
\hline $12: 00$ & 16,91 & 14,45 & 1,147 & 12,09 & 16,81 \\
\hline $13: 00$ & 16,44 & 14,57 & 1,135 & 12,23 & 16,91 \\
\hline $14: 00$ & 10,04 & 14,59 & 1,132 & 12,26 & 16,92 \\
\hline $15: 00$ & 12,85 & 14,49 & 1,136 & 12,15 & 16,83 \\
\hline $16: 00$ & 15,52 & 14,29 & 1,134 & 11,95 & 16,62 \\
\hline $17: 00$ & 18,46 & 13,98 & 1,123 & 11,67 & 16,30 \\
\hline $18: 00$ & 15,72 & 13,59 & 1,116 & 11,30 & 15,89 \\
\hline $19: 00$ & 12,52 & 13,14 & 1,123 & 10,83 & 15,46 \\
\hline $20: 00$ & 16,13 & 12,66 & 1,137 & 10,32 & 15,00 \\
\hline $21: 00$ & 11,35 & 12,18 & 1,140 & 9,83 & 14,52 \\
\hline $22: 00$ & 10,75 & 11,72 & 1,121 & 9,41 & 14,03 \\
\hline 23:00 & 13,91 & 11,32 & 1,081 & 9,10 & 13,55 \\
\hline
\end{tabular}

$$
\begin{aligned}
& m_{K_{2 U}}(t)=0,554-0,0026 t-0,036 \cos \frac{2 \pi t}{73}-0,025 \cos \frac{4 \pi t}{73}+0,0183 \cos \frac{6 \pi t}{73}+ \\
& +0,045 \sin \frac{2 \pi t}{73}+0,0397 \sin \frac{4 \pi t}{73}+0,0833 \sin \frac{6 \pi t}{73} ;
\end{aligned}
$$

коэффициент несимметрии напряжения по нулевой последовательности $\left(K_{0 U}\right)$ :

$$
\begin{aligned}
& m_{K_{0 U}}(t)=1,329+0,0024 t-0,0175 \cos \frac{2 \pi t}{73}+0,188 \cos \frac{4 \pi t}{73}+0,519 \cos \frac{6 \pi t}{73}+ \\
& +0,0069 \sin \frac{2 \pi t}{73}+0,0588 \sin \frac{4 \pi t}{73}-0,333 \sin \frac{6 \pi t}{73} .
\end{aligned}
$$

Проверка тренда на статическую значимость показала его 5\%-ный уровень.

Аналогично прогнозируются коэффициенты качества электроэнергии системы электроснабжения ИЖС на последующие годы с глубиной прогнозирования два года.

$$
-1086-
$$




\section{Выводы}

1. Проведенный статистический анализ коэффициентов качества электроэнергии позволил установить закономерность изменения коэффициентов в течение суток и подтвердил подверженность этих изменений случайным процессам с тремя характерными периодами. При этом получен набор выражений для определения наиболее вероятных значений показателей качества электроэнергии в характерные периоды суток с учетом ежегодного роста нагрузок в системах электроснабжения ИЖС на $5 \%$.

2. Учет вероятностного характера изменения показателей качества электроэнергии и их прогнозирование на ближайшие несколько лет позволит более обоснованно разрабатывать технические устройства и мероприятия по снижению негативного влияния несимметрии и несинусоидальности напряжения на энергетические показатели и на отдельные элементы системы электроснабжения ИЖС.

\section{Список литературы}

[1] Боярская Н.П., Довгун В.П., Егоров Д.Э. Синтез фильтрокомпенсирующих устройств для систем электроснабжения. Красноярск: СФУ, 2014, 192 с. [Boyarskaya N.P., Dovgun V.P., Egorov D.E. Synthesis filter-devices for power supply systems. Krasnoyarsk: SFU, 2014, 192 p. (in Russian)]

[2] Авербух М.А., Жилин Е.В. О потерях электроэнергии в системах электроснабжения индивидуального жилищного строительства. Энергетик, 2016, 6, 54-57 [Averbuch M.A., Zhilin E.V. About the loss of electricity in power systems for individual housing construction. Energetic, 2016, 6, 54-57 (in Russian)]

[3] Веников В.А. Электрические системы. Математические задачи электроэнергетики. Москва: Высшая школа, 1981. 288 с. [Venikov V.A. Electrical Systems. Mathematical problems of electric power industry, Moscow: Higher School, 1981. 288 p. (in Russian)]

[4] Авербух М.А., Забусов В.В., Пантелеев В.И. Системный подход к оценке параметров заземляющих сетей электроустановок северных промышленных комплексов. Красноярск: СФУ, 2009. 271 c. [Averbuch M.A., Zabusov V.V., Panteleev V.I. A systematic approach to the evaluation of the parameters of the grounding of electrical networks northern industrial complexes. Krasnoyarsk: SFU, 2009. 271 c. (in Russian)]

[5] Маталыцкий М., Хацкевич Г. Теория вероятностей, математическая статистика и случайные процессы. Минск: Высшая школа 2012. 720 с. [Matalytskii M., Hatckevich G. Probability theory, mathematical statistics and stochastic processes. Litres, 2016. 720 p. (in Russian)] 